\title{
Osteoporosis Treatment and Pathway Diagnosis Genetic Treatment
}

\section{Mahdi Bastani Pur Moghaddam*}

Dr.Orthopedic and Genetic,Outstanding researcher in USA and the United Kingdom

*Corresponding Author: Mahdi Bastani Pur Moghaddam, Dr.Orthopedic and Genetic,Outstanding researcher in USA and the United Kingdom. E-mail: mahdi.pur.moghaddam@gmail.com

Received: May 31, 2019; Published: July 08, 2019

DOI: $10.31080 /$ ASOR.2019.02.0075

\begin{abstract}
Osteoporosis is a major public health problem that is very common in the world and affects many people in the world, Its importance grows over time. Prevention of bone fracture and the treatment of osteoporosis can significantly reduce mortality and also significantly reduce direct and indirect economic costs. The most disappointing outcome of this disease is fracture.

Osteoporosis, in addition to being a silent disease, is the only symptom of osteoporosis, bone fracture, and there are no other serious symptoms before. BMD diagnostic devices (bone density) to diagnose you from your disease, There is, But you can identify your osteoporosis in a simple and homely way so you can know your condition before breaking your bone.
\end{abstract}

- If the vertical lines appear on your nail, and the more these lines become more prominent, your bone is vulnerable to fractures.

- If your nail is dry, your osteoporosis rate is high [1]

Keywords: Osteoporosis; Treatment

\section{Introduction}

We have discovered a gene, who develop your bone and skeletal growth from childhood.

This gene is due to osteoporosis-stimulating conditions, It may not work in our body or the gene's path may be destroyed.

We have designed a genetic medicine, Which suggests to our gene that you are a child, Then this gene begins its activity two times.

Be aware that this gene automatically makes the mineral you need for your bones and there is no need to use extra supplements.

The name is of the gene (homo) and the name is of our drug (hemotenici) [2].

\section{Treatment method}

Below the nail plate is a liquid that comes with some fat and protein, we extract this material from the patient's nail plate, in the liquid we have, there is the same hemo, in fact hemo exists in the genetic code.

Once we find the desired gene, we separate it, we have to do the hemo separation in this fluid. In fact, we one is designing a fluid that transmits the metabolism to the patient. And it should be in the central part of the skeleton that's near the neck inject.

\section{Period and manner of consumption}

To prevent 0.5 or $1 \mathrm{cc}$ per year and to treat 1.5 or $2 \mathrm{cc}$ depending on the stage of the disease is enough.

We even know how to increase this substance, and this does not require any re-manufacturing of medicine during treatment [3].

\section{More about Hemo}

Hemo is a genetic substance, which exists in the wall of the fetal sac, and plays an important role in making the baby's skeleton.

When the fetus develops, this material is stored as a bone support on the nail plate.

The insignificant effect of osteoporosis drugs and supplements

Sense of taste

The responsibility of this sense is to give a message to the brain, this message includes the diagnosis of the material you're eating.

\section{What is material recognition?}

That is, the message carries information about the presence of minerals and vitamins in the feed. 
The brain reads this message, Identifies the material the body needs, And for the required materials. The brain, to allow the material to enter the mainstream of the body.

When you take a vitamin tablet the acts mentioned above are not happening. Because the tablets, or are laminated, are consumed in capsule form, that your body does not recognize it. And this will make a lot of these vitamins repel and a small amount of it is absorbed through the intestine.

\section{Conclusion}

We conclude that these drugs do not have much effect on the recovery of osteoporosis.

The importance of gene therapy

The main principle of this treatment is to introduce external genetic material to modify or change the cell function. Genetic materials, which are known today as gene therapy, can be used to treat osteoporosis by genetic engineering techniques.

\section{Advantages of gene therapy}

The benefits of gene therapy for future patients can significantly help the quality of life of the elderly and restores the lives of patients.

\section{Bibliography}

1. Mahdi Bastani Pur Moghaddam. "A Definitive Prevention and Treatment of Osteoporosis through Genetic Therapy". EC Orthopaedics 10.3 (2019): 159-161.

2. Mahdi Bastani Pur Moghaddam. "The Importance and Resuscitation of Osteoporosis patients through Genetic Therapy". EC Orthopaedics 10.6 (2019): 369-370.

3. M Bastani Pur Moghadam. Journal of Clinical \& Experimental Orthopaedics 4 (2018).

\section{Volume 2 Issue 8 August 2019}

(C) All rights are reserved by Mahdi Bastani Pur Moghaddam. 